\title{
Hyperammonemia from a Urea Cycle Disorder Presenting in Adulthood
}

\author{
Tiffany C. Priester ${ }^{1}$, Teck-Kim Khoo ${ }^{1,2}$, Evans R. Fernández-Pérez ${ }^{1,3}$, Kevin R. Regner ${ }^{1,4}$, \\ Jennifer A. Tracy ${ }^{5}$, Sabrina Mitchell ${ }^{6}$, Marshall L. Summar ${ }^{6}$ and Dusica Babovic-Vuksanovic ${ }^{*}, 7$ \\ ${ }^{I}$ Department of Internal Medicine, Mayo Clinic, Rochester, USA \\ ${ }^{2}$ Division of Endocrinology, Nutrition and Metabolism, Mayo Clinic, Rochester, USA \\ ${ }^{3}$ Pulmonary and Critical Care Medicine, Mayo Clinic, Rochester, USA \\ ${ }^{4}$ Division of Nephrology and Hypertension, Mayo Clinic, Rochester, USA \\ ${ }^{5}$ Department of Neurology, Mayo Clinic, Rochester, USA \\ ${ }^{6}$ Center for Human Genetic Research and Division of Medical Genetics, Vanderbilt University, USA \\ ${ }^{7}$ Department of Medical Genetics, Mayo Clinic, Rochester, USA
}

\begin{abstract}
OBJECTIVE: The aim of this report is to describe a patient with late presentation of carbamyl phosphate synthetase I (CPS-I, EC 6.3.4.16) deficiency, a rare urea cycle deficiency, and to facilitate recognition and treatment of patients presenting with encephalopathy and hyperammonemia in a critical care setting. DESIGN: Case Report. SETTING: Intensive care unit of Saint Mary's Hospital, Mayo Clinic, Rochester, Minnesota. PATIENT: A 65-year-old woman admitted with progressive encephalopathy. INTERVENTIONS: Intubation and mechanical ventilation, protein-restricted parenteral nutrition, intravenous arginine, hemodiafiltration, and intravenous antibiotic therapy. MEASUREMENTS AND MAIN RESULTS: Serum ammonia and glutamine levels were elevated, but other laboratory and imaging investigations were unremarkable. Despite the above interventions, her mental status deteriorated. She developed ventilator associated pneumonia, which worsened despite antibiotic treatment. The family decided to withdraw care and the patient expired on hospital day 10. A postmortem enzyme assay on fresh-frozen liver tissue showed severely diminished CPS-I activity. CONCLUSIONS: To our knowledge, this is the oldest reported age at presentation of CPS-I deficiency. Urea cycle disorders should be part of the differential diagnosis of hyperammonemia regardless of age as early treatment may ameliorate mortality and morbidity in these patients.
\end{abstract}

\section{INTRODUCTION}

Urea cycle enzymatic deficiencies are rare inherited conditions that typically present in a newborn's first days of life when they begin to receive enteral protein [1]. However, partial enzyme deficiencies may present in adolescence and adulthood [2]. Onset may be triggered with any stress resulting in acute catabolic state, such as surgery or childbirth, or use of valproic acid, as reported in several cases [3-5].

Dietary protein is broken down into nitrogen, among other byproducts, and is then excreted as urea via the urea cycle (Fig. 1) through a series of enzymatic conversions in the liver. Carbamyl phosphate synthetase-I (CPS-I, EC 6.3.4.16) is the rate-limiting enzyme that catalyzes the first step of the hepatic urea cycle. The prevalence of CPS-I deficiency is estimated at one in 800,000 to $1,000,000$. Both CPS-I deficiency and ornithine transcarbamylase (OTC) deficiency usually present with hyperammonemia in early age, but delayed onset of metabolic decompensation is possible. Call et al. previously described the case of a 33-year-old

*Address correspondence to this author at the Mayo Clinic, Department of Medical Genetics, $2001^{\text {st }}$ St SW, Rochester MN 55905, USA; Tel: 507-2662967; Fax: 507-284-1067; E-mail: dbabovic@mayo.edu woman diagnosed with CPS-I deficiency [6]. We present a case of CPS-I deficiency in a 65-year-old woman with the encephalopathy, hyperammonemia, and respiratory alkalosis. To our knowledge, this is the oldest age of presentation reported.

\section{CASE PRESENTATION}

A 65-year-old woman was transferred to our facility for severe progressive encephalopathy soon after uneventful abdominal hernia repair surgery. Her past medical history was significant for several normal pregnancies and deliveries of healthy children, a back surgery, and two episodes of abdominal pain associated with vomiting which were assumed to be pancreatitis. The patient was then diagnosed with abdominal hernia and underwent surgery. Two days after the procedure, her mental status began to progressively decline and she was transferred to Mayo Clinic for further care. On presentation to our Emergency Department, her Glasgow Coma Score was 3 and she was endotracheally intubated for airway protection. There was no localizing feature on her neurologic exam. Her serum ammonia level was $140 \mu \mathrm{Mol} / \mathrm{L}$ (reference range: < 50) on admission and increased to 218 $\mu \mathrm{Mol} / \mathrm{L}$ the following day (see Fig. 2). Arterial blood gas analysis showed a persistent respiratory alkalosis while breathing spontaneously. There was no significant anion gap. 


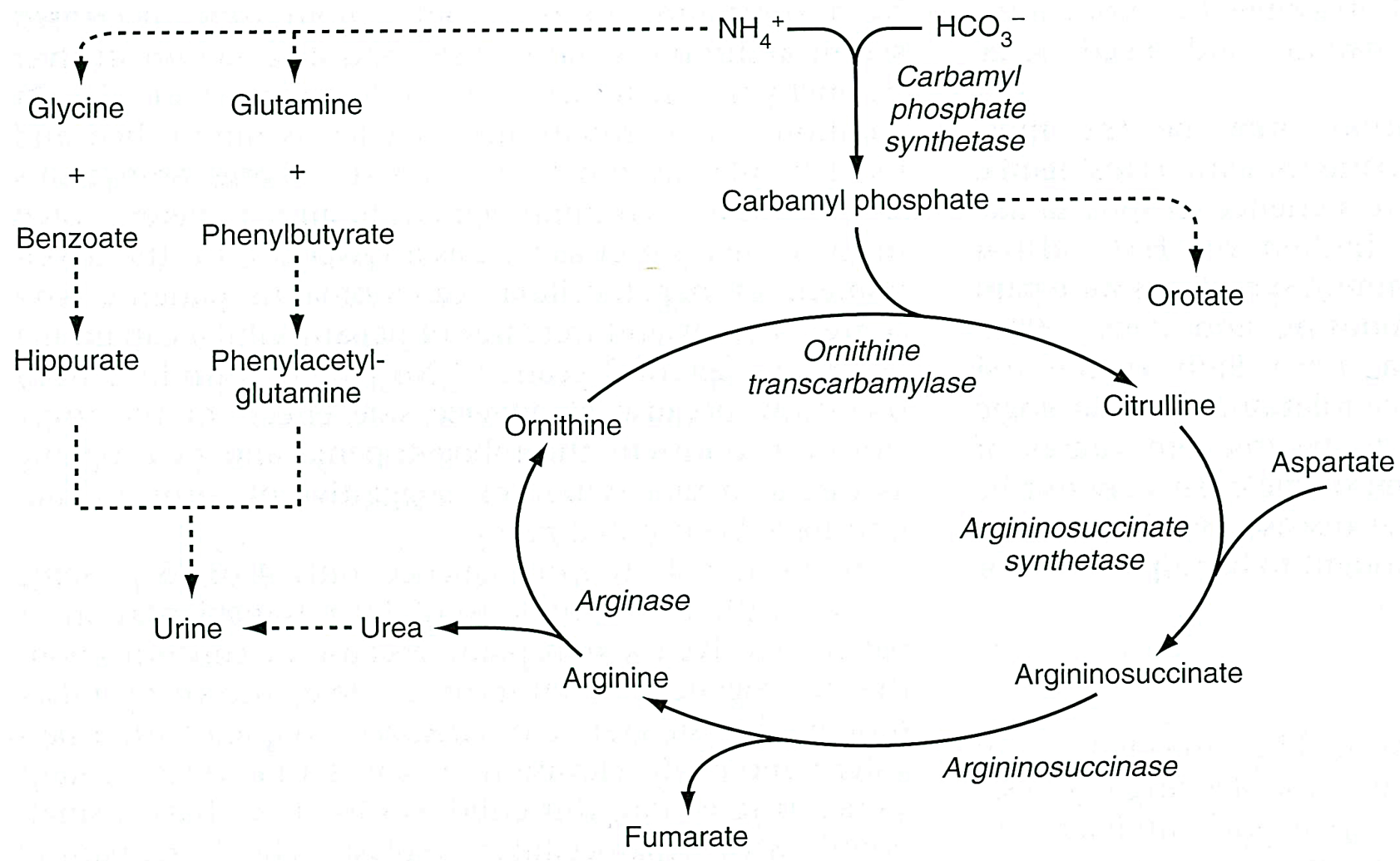

Fig. (1). The urea cycle with accessory pathway. Ammonia is generated during protein catabolism, converted to urea in the liver, and secreted by the kidneys. Excess ammonia may be converted into a form secreted by the kidneys via an accessory pathway, as illustrated to the left in the figure. (Reprinted from Figure 73-5 from Feldman: SLEISENGER \& FORDTRAN'S GASTROINTESTINAL AND LIVER DISEASE, pg 1626, 8/E (C) 2006 Elsevier Inc, reproduced with permission from Elsevier, Inc.).

Other laboratory studies performed were normal, including liver transaminases, electrolytes, creatinine, urinalysis and routine spinal fluid analysis. Computed tomography (CT) of the head was unremarkable and CT of the abdomen and pelvis demonstrated only mild fatty changes of the liver. We were unable to perform an MRI as she had metallic surgical clips in her neck from a previous surgery. EEG showed severe slowing and decreased amplitude of background activity with intermittent generalized triphasic waves.

\section{Plasma Ammonia Levels}

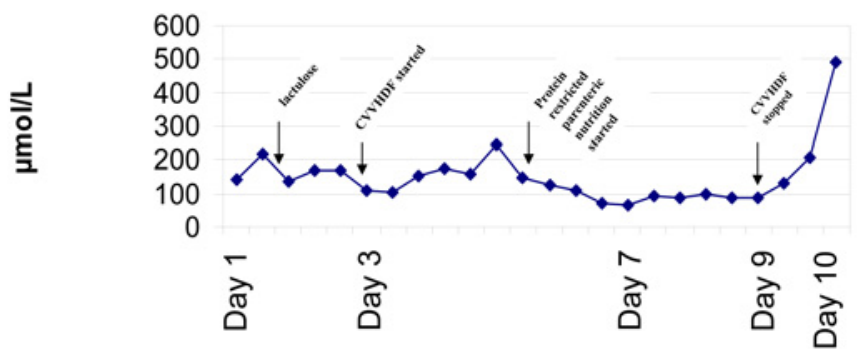

Fig. (2). Trend of plasma ammonia levels. Ammonia levels improved with initiation of continuous venovenous hemodiafiltration (CVVHDF) but increased after development of sepsis. The lowest plasma levels of ammonia were achieved with combination of CVVHDF and protein-restricted parenteral nutrition. Ammonia levels increased dramatically after CVVHDF discontinuation.
Given her presentation with hyperammonemia, decreased consciousness, and respiratory alkalosis without generalized liver dysfunction, a urea cycle disorder was suspected. The initial serum amino acid panel demonstrated extremely high plasma glutamine levels $(5531 \mu \mathrm{Mol} / \mathrm{L}$; reference range: 205-756 $\mu \mathrm{Mol} / \mathrm{L}$ ), but normal levels of citrulline (39 $\mu \mathrm{Mol} / \mathrm{L}$; reference range: $12-55 \mu \mathrm{Mol} / \mathrm{L})$ and arginine $(38$ $\mu \mathrm{Mol} / \mathrm{L}$; reference range: $15-128 \mu \mathrm{Mol} / \mathrm{L})$. An urine orotic acid level was found to be slightly elevated (15.8 $\mathrm{mmol} / \mathrm{mmol}$ creatinine; reference range: $0.4-1.2 \mathrm{mmol} / \mathrm{mmol}$ creatinine). However, prior to sample collection, the patient was on long-term treatment with allopurinol for a previous history of gout, therefore interpretation of the urine orotic acid level was difficult.

Due to persistent hyperammonemia and encephalopathy, treatment with lactulose, continuous venovenous hemodiafiltration (CVVHDF), low-protein parenteral nutrition, and intravenous arginine was initiated. Her ammonia level decreased to $64 \mu \mathrm{Mol} / \mathrm{L}$ (see Fig. 2), but she remained unconscious with no improvement in mental status. The patient subsequently developed a ventilator-associated pneumonia with growth of methicillin-sensitive Staphylococcus aureus from bronchoalveolar lavage fluid. Given lack of clinical improvement, the family withdrew therapy on day 9 of her hospital stay. Her ammonia increased to a peak of 490 $\mu \mathrm{Mol} / \mathrm{L}$ and she expired on day 10 .

A transcutaneous needle liver biopsy for enzyme analysis was obtained minutes after her death. Fresh frozen hepatic 
tissue was sent to the Children's National Medical Center, Washington, D.C., where enzyme assays were performed for OTC and CPS-I activity. Results showed markedly reduced CPS-I activity that measured only $4.9 \%$ of control. The OTC activity was substantial, although slightly below control.

Molecular analysis including SSCP and gene sequencing was performed at the center for Human Genetic Research at Vanderbilt University Medical Center. SSCP analysis was done with 43 pairs of primers spanning each of the CPS-I exons and the 3'UTR. Two novel changes were identified in the CPS-I gene. First, there was a homozygous $\mathrm{C} \rightarrow \mathrm{G}$ substitution at nucleotide 3618 in exon 29, resulting in a synonymous change (both GTC and GTG code for Valine). Therefore, the significance of this substitution remains unclear. The possibility that it acts as an exon splice enhancer could not be assessed. Second, there was a heterozygous $G$ $\rightarrow$ A change in intron 28, located 25 bases from the start of Exon 29. Considering the position of the change, it is difficult to conclude that this was a pathogenic alteration.

The family history was negative for metabolic abnormalities, cognitive deficiencies, neurologic disorders, and there were no neonatal or childhood deaths. The patient did not have similar episodes in the past. Her dietary history was characterized by avoidance of high-protein foods throughout the lifetime, especially during times of illness or high stress. While awaiting definitive diagnosis, X-linked OTC deficiency was considered since this is the most common urea cycle defect. An allopurinol challenge was offered to both her daughters, and the result was negative (urine orotic acid levels after a dose of allopurinol were normal).

\section{DISCUSSION}

The most common urea cycle defect is OTC deficiency, an X-linked disorder. All other urea cycle defects, including CPS-I deficiency, are inherited in an autosomal recessive fashion. Most patients present in infancy with seizures, irritability, and encephalopathy prompting medical investigation. When the diagnosis is made after the neonatal period, clinical features typically include nausea and vomiting, somnolence, seizures, and coma [2-6], but more subtle neurobehavioral disturbance and cognitive slowing are often dominant clinical features. Partial enzyme deficiencies may have delayed presentation as in our case. Therefore a high level of suspicion for inherited urea cycle disorders should be maintained in the context of a hyperammonemic crisis at any age. Undiagnosed and untreated patients suffer significant neurocognitive damage and therefore early diagnosis and intervention are paramount. In some patients the hyperammonemia and frequency of hyperammonemic crises can be reduced or avoided with a protein restricted diet and arginine supplementation [7, 8]. Predisposing circumstances that may lead to a hyperammonemic crisis include high protein intake, post-surgical state, child birth, and any process that induces a catabolic state and excess protein breakdown [4, 9]. Use of valproic acid, which inhibits the synthesis of urea, has also been reported to precipitate a crisis $[3,4,10]$.

With advanced diagnostics, the adult onset of metabolic conditions is more frequently recognized, particularly in individuals with partial enzyme deficiency. Therefore, identification and treatment of these conditions, including CPS-I deficiency, is of increasing importance to internists and other practitioners of adult health care. Treatment in the critical care setting is directed at acutely decreasing ammonia levels. A number of extracorporeal modalities including exchange transfusion, peritoneal dialysis, hemofiltration, and hemodialysis have been reported to reduce ammonia levels in patients with urea cycle disorders [7, 11, 12 ]. We chose to dialyze our patient using CVVHDF to allow for continuous ammonia clearance and to avoid hemodynamic instability. Management of both acute hyperammonemic crisis and chronic hyperammonemia involves protein restriction and supplemental arginine along with ammonia conjugating agents that enhance nitrogen excretion while bypassing the urea cycle (Fig. 1). During acute crisis, a loading dose of $55 \mathrm{~mL} / \mathrm{m}^{2}$ of $10 \%$ sodium benzoate/sodium phenylacetate with $2.0 \mathrm{~mL} / \mathrm{kg}$ arginine hydrochloride solution in $25 \mathrm{~mL} / \mathrm{kg}$ of $10 \%$ dextrose infused over 90 minutes have been recommended for adults [13]. The solution can then be infused over 24 hours continuously until the patient can take oral medications. Electrolytes should be checked frequently as potassium can be depleted by treatment and should be included in maintenance fluids and replaced as needed. Onehalf or one-quarter normal saline is recommended for maintenance fluids given the high sodium and chloride load in the treatment solution. Dietary protein should be avoided for the first 24 hours and then should be resumed enterally or parenterally with guidance from a nutrition specialist [13]. Additionally, avoidance of a catabolic state with appropriate amounts of non-protein caloric nutrition should be started early in order to prevent build-up of serum ammonia from internal sources [13-15]. Due to inability to generate arginine, patients with CPS-I deficiency require arginine supplementation on a chronic basis. As the enzymes involved in the urea cycle are predominantly found in liver mitochondria, successful treatment with orthotopic liver transplantation has also been described in the literature $[12,16]$.

Neurologic symptoms have been linked to elevated ammonia levels and cerebral injury during hyperammonemic crisis. Several proposed mechanisms include cerebral edema, direct neurotoxicity, and astrocyte dysfunction. It has been suggested that glutamine accumulation causes alterations in neurotransmitter metabolism and cerebral swelling. In astrocytes, excess ammonia is conjugated to glutamate by glutamine synthetase generating glutamine. Although this process is initially protective, glutamine is neurotoxic at high concentrations. In addition, ammonia directly activates $\mathrm{N}$ methyl-D-aspartate (NMDA) receptors leading to neurotoxicity and irreversible brain damage [17]. Cell damage and edema in the brain can be detected by magnetic resonance imaging (MRI) [18], although this modality cannot distinguish reversible from irreversible damage.

Unlike some other individuals with adult presentation of urea cycle disorder, our patient had very few symptoms with previous surgery and pregnancies. Family did not report reduced cognitive function previously. However, history of life long protein aversion and recurrent vomiting in recent past (probably wrongly attributed to "pancreatitis") are suspicious of unrecognized mild hyperammonemia in this patient. It is not clear why the patient developed such a dramatic clinical course after abdominal surgery, but possibility of mild hyperammonemia before anesthesia, coupled with increased catabolic state related to procedure, possible protein load due to intraoperative albumin administration, can not be ex- 
cluded as contributing factors. There was no evidence of other hepatic injury on liver biopsy and there was no evidence of viral hepatitis. There was no evidence of sepsis prior to her hyperammonemic crisis.

The preliminary diagnosis of CPS-I deficiency is based on biochemical findings of hyperammonemia and elevated glutamine in the setting of normal urinary orotic acid; confirmatory diagnosis is performed by enzyme assay on freshfrozen liver tissue. The lack of family history of hyperammonemia is not surprising, given that CPS-I deficiency is an autosomal recessive condition. Although we were not able to confirm that DNA variants in our patient are pathogenic, all biochemical evaluations and enzymatic studies are supportive of partial CPS-I deficiency. Similarly, gene sequencing will only detect $70 \%$ of patients with OTC enzyme deficiency [19].

Prognosis for neurocognitive outcome in children with urea cycle disorders is often uncertain, but ammonia level at the time of diagnosis appears to be predictive of permanent adverse neurologic effects [20]. The outcomes of patients who present in adulthood with their first hyperammonemic episode is even less clear. Unfortunately, the outcome in our patient was poor, probably due to delayed diagnosis of urea cycle defect and late start of treatment of hyperammonemia. As early recognition and timely treatment of urea cycle disorders may reduce morbidity and mortality, they should be part of the differential diagnosis of isolated hyperammonemia in adult as well as pediatric medicine. Early detection, accurate diagnosis, and implementation of appropriate treatment strategies may be of benefit in CPS-I deficiency before irreversible brain injury develops.

\section{ACKNOWLEDGEMENTS}

We are grateful for the secretarial support provided by Mrs. Cheryl A. Dowse in completing the manuscript. We also thank Mr. Nikola Babovic for his helpful editorial comments.

\section{REFERENCES}

[1] Lyon G, Adams R, Kolodny E. The neurology of neonatal hereditary metabolic diseases. Neurology of hereditary metabolic diseases of children. 2nd ed. New York: McGraw-Hill; 1996: pp. 6-44.

[2] Legras A, Labarthe F, Maillot F, et al. Late diagnosis of orthinthine transcarbamylase defect in three related female patients: polymorphic presentations. Crit Care Med 2002; 30: 241-4.
[3] Verbiest H, Straver J, Colombo J, van der Vijver J, van Woerkom T. Carbamyl phosphate synthetase-1 deficiency discovered after valproic acid-induced coma. Acta Neurol Scand 1992; 86: 275-9.

[4] Leao M. Valproate as a cause of hyperammonemia in heterozygotes with ornithine-transcarbamylase deficiency. Neurology 1995; 45: 593-4.

[5] Arn P, Hauser E, Thomas G, et al. Hyperammonemia in women with a mutation at the ornithine carbamoyltransferase locus: a cause of postpartum coma. N Engl J Med 1990; 322: 1652-5.

[6] Call G, Seay A, Sherry R, Qureshi I. Clinical features of carbamyl phosphate synthetase-I deficiency in an adult. Ann Neurol 1984; 16: $90-3$.

[7] Wiegand C, Thompson T, Bock G, et al. The management of lifethreatening hyperammonemia: a comparison of several therapeutic modalities. J Pediatr 1980; 96: 142-4.

[8] Nicolaides P, Liebsch D, Dale N, Leonard J, Surtees R. Neurological outcomes of patients with ornithine carbamoyltransferase deficiency. Arch Dis Child 2002; 86: 54-6.

[9] Wong L, Craigen W, O'Brien W. Postpartum coma and death due to carbamoyl-phosphate synthetase I deficiency. Ann of Int Med 1994; 120: 216-7.

[10] Takeoka M, Soman T, Shih V, Caviness VJ, Krishnamoorthy K. Carbamyl phosphate synthetase I deficiency: a destructive encephalopathy. Pediatr Neurol 2001; 24: 193-9.

[11] Summar M, Pietsch J, Deshpande J, Schulman G. Effective hemodialysis and hemofiltration driven by an extracorporeal membrane oxygenation pump in infants with hyperammonemia. J Pediatr 1996; 128: 379-82.

[12] Todo S, Starzl T, Tzakis A, et al. Orthotopic liver transplantation for urea cycle enzyme deficiency. Hepatology 1992; 15: 419-22.

[13] Summar M. Current strategies for the management of neonatal urea cycle disorders. J Pediatr 2001; 138: S30-9.

[14] Brusilow S. Arginine, an indispensable amino acid in patients with inborn errors of urea synthesis. J Clin Invest 1984a; 74: 2144-8.

[15] Wong K, Wong S, Lam S, Tam S, Tsoi N. Ammonia clearance by peritoneal dialysis and continuous arteriovenous hemodiafiltration. Pediatr Nephrol 1998; 12: 589-91.

[16] Zimmer K, Naim H, Koch H, et al. Survival after early treatment for carbamyl phosphate synthetase (CPS) I deficiency associated with increase of intramitochondrial CPS I. Lancet 1995; 346: 15301.

[17] Brusilow S, Danney M, Waber L, et al. Treatment of episodic hyperammonemia in children with inborn errors of urea synthesis. N Engl J Med 1984b; 310: 1630-4.

[18] Cooper A. Role of glutamine in cerebral nitrogen metabolism and ammonia neurotoxicity. Ment Retard Dev Disabil Res Rev 2001; 7 : 280-6.

[19] Scaglia F, Zheng Q, O'Brien W, et al. An integrated approach to the diagnosis and prospective management of partial ornithine transcarbamylase deficiency. Pediatrics 2002; 109: 150-2.

[20] Bachman C. Outcome and survival of 88 patients with urea cycle disorders: a retrospective evaluation. Eur J Pediatr 2003; 162: 4106. 